\title{
Respiratory viruses associated with acute wheezing in hospitalized young children in Jordan
}

Olajumoke Fadugba ${ }^{1}$, Zaid Haddadin ${ }^{2}$, Sylvie Muhimpundu ${ }^{2}$, Samir Faouri ${ }^{3}$, Asem Shehabi $^{4}$, Herdi Rahman ${ }^{2}$, John Williams ${ }^{5}$, E. Kathryn Miller ${ }^{6}$, Najwa Khuri-Bulos ${ }^{4}$, and Natasha Halasa ${ }^{2}$

${ }^{1}$ University of Pennsylvania Perelman School of Medicine

${ }^{2}$ Vanderbilt University Medical Center

${ }^{3}$ Al-Bashir Hospital

${ }^{4}$ The University of Jordan

${ }^{5}$ University of Pittsburgh

${ }^{6}$ Vanderbilt University

October 5, 2020

\begin{abstract}
Background Viral acute respiratory infections (ARI), including respiratory syncytial virus (RSV) and human rhinovirus (HRV), induce acute wheezing episodes in young children. This study aimed to determine the association of wheezing with respiratory viruses, demographic and clinical characteristics in young children. Methods A prospective viral surveillance study in children less than two years, admitted with fever and/or respiratory symptoms in Amman, Jordan, 2010-2013. Demographic and clinical characteristics were collected through parent interviews and medical chart abstraction. Nasal/throat swabs were collected and tested by qRT-PCR. Multivariable logistic regression was fit to assess the association between wheezing and a-priori selected predictors of interest. Results Of 3168 children enrolled, 1757 (55\%) were wheezing-positive, who were older, more likely to have a respiratory virus, underlying medical conditions, personal history of reactive airway disease (RAD), and a family history of asthma, compared to wheezing-negative children. Older age, personal history of RAD, family history of asthma, RSV and HMPV were associated with higher odds of wheezing in an adjusted regression model. Conclusion In young children, RSV and HMPV were associated with higher odds of wheezing. Longitudinal studies are needed to evaluate the association between early childhood viral ARI and recurrent wheezing later in childhood, to guide preventive interventions.
\end{abstract}

\section{Conflicts of interest}

Natasha Halasa, MD, MPH receives grant support from Sanofi, Quidel, and speaker compensation from an education grant supported by Genentech. Sanofi also donated vaccines and influenza antibody testing for influenza vaccine trial. John Williams, MD is on the scientific board for Quidel, Independent Data Safely Monitoring Committee, GlaxoSmithKline, scientific advisory board ID Connect. All the other authors have no conflicts of interest to disclose.

\section{Funding}

This work was supported by the UBS Optimus Foundation; National Institutes of Health and the CTSA award UL1TR000445 from the National Center for Advancing Translational Sciences. Its contents are solely the responsibility of the authors and do not necessarily represent official views of the National Center for Advancing Translational Sciences or the National Institutes of Health.

\section{Abstract}




\section{Background}

Viral acute respiratory infections (ARI), including respiratory syncytial virus (RSV) and human rhinovirus (HRV) induce acute wheezing episodes in young children. This study aimed to determine the association of wheezing with respiratory viruses, demographic and clinical characteristics in young children.

\section{Methods}

A prospective viral surveillance study in children less than two years, admitted with fever and/or respiratory symptoms in Amman, Jordan, 2010-2013. Demographic and clinical characteristics were collected through parent interviews and medical chart abstraction. Nasal/throat swabs were collected and tested by qRTPCR. Multivariable logistic regression was fit to assess the association between wheezing anda-priori selected predictors of interest.

\section{Results}

Of 3168 children enrolled, $1757(55 \%)$ were wheezing-positive, who were older, more likely to have a respiratory virus, underlying medical conditions, personal history of reactive airway disease (RAD), and a family history of asthma, compared to wheezing-negative children. Older age, personal history of RAD, family history of asthma, RSV and HMPV were associated with higher odds of wheezing in an adjusted regression model.

\section{Conclusion}

In young children, RSV and HMPV were associated with higher odds of wheezing. Longitudinal studies are needed to evaluate the association between early childhood viral ARI and recurrent wheezing later in childhood, to guide preventive interventions.

\section{Key Messages}

In children, less than two years, RSV and HMPV, but not HRV, were associated with wheezing. Future studies are needed to clearly determine which virus interventions would be most effective at preventing the development of wheezing illness.

\section{Background}

Wheezing is a continuous musical sound that can be produced by oscillation of opposing walls of an airway that is narrowed almost to the point of closure ${ }^{1}$; it affects approximately one-third of children at least once by the age of nine and is a significant cause of morbidity and mortality in young children worldwide ${ }^{2,3}$. While wheezing is only transient for most children, around $40 \%$ are still diagnosed with recurrent wheezing or asthma after the age of six years ${ }^{2,4}$. Wheezing is often induced by various respiratory tract infections, which include respiratory syncytial virus (RSV), human rhinovirus (HRV), human metapneumovirus (HMPV), influenza viruses, parainfluenza viruses (PIV), and coronavirus ${ }^{2}$. While RSV is the leading cause of hospitalization for lower respiratory tract infection (LRTI) and first wheezing episode in infants ${ }^{3,5}$, recent improvements in virus detection through methods such as real-time reverse transcriptase polymerase chain reaction (qRT-PCR) have allowed investigators to better elucidate the important role of HRV in LRTIs, wheezing and asthma development. HRV is now known to be the most common virus associated with wheezing at ages 6-12 months and is the second most common virus detected among wheezing infants during the first six months of life, after $\mathrm{RSV}^{6,7}$.

Developments in molecular diagnostics have also contributed to the discovery of HMPV and its role in respiratory illnesses ${ }^{8}$. Because studies show that virus-associated wheezing and LRTI during infancy are associated with the subsequent development of childhood asthma, it is important to determine which viruses are most strongly associated with wheezing and to assess other risk factors that contribute to wheezing, in order to develop interventions when possible ${ }^{6}$. Few viral epidemiological studies in the Middle East focus on the association between wheezing and respiratory viruses ${ }^{9,10}$. Our study aimed to determine the association of wheezing with respiratory viruses, demographic and clinical characteristics in young children. 


\section{Methods}

\section{Study design and population}

This was a sub-study of our main prospective viral surveillance study of hospitalized children at a large government hospital, Al Bashir, in Amman, Jordan ${ }^{11}$. Subjects were enrolled five days a week between March 16, 2010 and March 31, 2013 within 48 hours of hospital admission. Eligible subjects were children less than two years admitted to the hospital with fever and/or respiratory symptoms plus one or more of the following admission diagnoses: acute respiratory illness, asthma exacerbation, bronchiolitis, bronchopneumonia, croup, pneumonia, pneumonitis, pertussis or pertussis-like cough, upper respiratory infection, cystic fibrosis exacerbation, respiratory distress, apnea, febrile seizure, fever without localizing signs, and sepsis rule-out. Children were excluded if they had fever with neutropenia, or if they were newborns who were never discharged from the hospital. This study was approved by the Institutional Review Boards at Vanderbilt University Medical Center (VUMC), Jordan University and the Jordanian Ministry of Health. Written informed consent was obtained from parents or guardians of all participants.

\section{Demographic and clinical information}

Study personnel completed case report forms by interviewing parents or guardians and extracting clinical information from medical records, including radiographic and laboratory data. Demographic and clinical information collected included: underlying medical conditions (UMCs), gestational age, birth weight, mode of birth delivery [Caesarean section (C-section) or normal spontaneous vaginal delivery (NSVD)], child's medical history including vaccination history, feeding, including supplemental vitamins, atopic history (asthma, allergic rhinitis or eczema) using the International Study of Asthma and Allergies in Childhood questionnaire ${ }^{12}$, family history of asthma, allergic rhinitis or eczema, current or history of breastfeeding, number of siblings, day care attendance, indoor pets, and household smoke exposure. Smoke exposure includes cigarette smoke and nargila, a form of smoking traditionally found in Middle Eastern cultures, in which tobacco is heated with charcoal and the resultant smoke is passed through a water basin before inhalation ${ }^{13}$. Subjects were considered to have UMCs if they had any of the following conditions: diabetes, heart disease, Down syndrome, kidney disease, sickle cell disease, cystic fibrosis, cancer, genetic/metabolic, cerebral palsy, neurological, mental retardation/developmental delay, seizure disorder, chronic diarrhoea (eg, $>2$ weeks), gastro-oesophageal reflux disease, immunodeficiency, asthma/reactive airway disease (RAD), liver disease and/or other. Data were entered into a standardized, secured database - REDCap (Research Electronic Data Capture) system (www.project-redcap.org) ${ }^{14}$.

\section{Diagnosis}

The treating physician prospectively recorded physical exam findings, including wheezing, and the admission diagnosis on a standardized case report form. Information regarding illness severity was also collected including length of hospital stay, intensive care unit (ICU) admission, supplemental oxygen requirement, and mechanical ventilation.

\section{Specimen collection and viral testing}

Nasal and throat swabs were collected from hospitalized infants and combined into transport medium (M4RT ${ }^{\circledR}$, Remel), aliquoted into MagMAX ${ }^{\mathrm{TM}}$ Lysis/Binding Solution Concentrate (Life Technologies), snap frozen, and stored at $-80^{\circ} \mathrm{C}$. Original and lysis buffer aliquots were shipped to VUMC on dry ice and were tested using qRT-PCR assays directed at primers and probes as previously published for the following respiratory viruses: $\mathrm{RSV}^{15}, \mathrm{HRV}^{16}, \mathrm{HMPV}^{17}$, influenza $\mathrm{A}$ and $\mathrm{B}^{18}$, PIV 1, 2 and 3, adenovirus, and Middle East respiratory syndrome coronavirus (MERS-CoV) ${ }^{19}$.

\section{Statistical analysis}

Summary statistics were presented as median with interquartile range (IQR) or percentage where appropriate. Wilcoxon rank sum test or Pearson Chi-square test were used to compare variables between wheezing and non-wheezing patients. The 14 tests were Bonferroni-corrected and interpreted based on Type I error rate of 
$\alpha=0.0036$. To assess the association between demographic and clinical characteristics, and viruses with the primary outcome of interest (i.e., wheezing), multivariable logistic regression was used to determine the odds ratios (OR) for wheezing across a-priori selected predictors of interest, including: age, sex, smoke exposure, breastfeeding history, birthweight, type of delivery, prematurity, personal, mother and family history of asthma, and virus type. All analyses had a significance level of 0.05 (two-tailed) and were performed using StataCorp, College Station, TX software (version 15.1).

\section{Results}

\section{Clinical and demographic characteristics}

During the study period, 3793 patients were eligible and 3168 subjects $(83.5 \%)$ were included in the final cohort (Figure 1). The median age at enrollment was 3.5 months, 1912 subjects (60\%) were male, median birth weight was 3 kilograms, median gestational age was 40 weeks, and $28 \%$ of children were delivered by C-section ${ }^{11}$. Most subjects ( $77 \%$ ) had exposure to household smoke, $84 \%$ were breastfed, and $2 \%$ attended daycare. Fourteen percent of subjects had an atopic mother with asthma, allergic rhinitis, or eczema, $3 \%$ had maternal asthma, $23 \%$ had a family history of asthma, and $6 \%$ had a personal history of atopy. Median length of hospital stay was 5 days, 1013 subjects $(32 \%)$ received oxygen supplementation during hospitalization, $284(9 \%)$ were admitted to the ICU and $111(4 \%)$ were on mechanical ventilation during hospitalization 11. Overall, $2581(81.5 \%)$ had at least one-virus detected, with $944 / 2581(37 \%)$ having at least one virus codetected, and 1757 subjects (55\%) had wheezing diagnosed by a physician (Figure 1).

\section{Wheezing and non-wheezing subjects}

On univariable analysis, children with wheezing were older; more likely to have UMCs; personal history of RAD, allergic rhinitis, and atopy; family history of asthma; receive oxygen; have a shorter LOS, but less likely to have a history of breastfeeding compared to non-wheezing subjects (Table 1). There were no differences in smoke exposure, prematurity, maternal history of atopy or ICU admission between the two groups (Table 1).

At least one virus was detected in $2581(81 \%)$ of all subjects. In the wheezing group, viruses were detected in $1540(88 \%)$ subjects, compared to $1041(74 \%)$ in the non-wheezing group $(\mathrm{p}<0.005)$ (Figures 1). Figure 2 illustrates compared to non-wheezing subjects, those with wheezing had a higher frequency of RSV ( $53 \%$ vs. $33 \%)$, HMPV (11\% vs. $6 \%$ ), and any virus( $88 \%$ vs. $74 \%)$. There was no difference in HRV detection between wheezing and non-wheezing subjects.

Virus detection by age among virus-positive wheezing subjects

RSV was detected more frequently in younger subjects than older subjects; $78 \%$ of patients [?] 2 months old had RSV compared with $41 \%$ of those aged 12-24 months $(\mathbf{p}<\mathbf{0 . 0 0 1}$; Figure 3). In contrast, AdV, HMPV, and flu were detected more frequently in older versus younger patients (Figure 3).

Effect of viruses and clinical/demographic factors on wheezing

On multivariable logistic regression model, older age (OR:1.082; 95\% CI: 1.064-1.100), RSV (OR:2.726; 95\% CI: 2.324-3.196), HMPV (OR:2.330; 95\% CI: 1.729-3.138), family history of asthma (OR:1.231; 95\% CI: 1.029-1.473), and personal history of RAD (OR:3.556; 95\% CI: 1.808-6.994) were associated with increased odds of wheezing(Figure 4). No association between wheezing and other clinical or demographic factors including sex, gestational age, birth weight, prematurity, delivery method, smoke exposure, breastfeeding, maternal asthma, flu, PIV, AdV or HRV ( $\mathrm{p}>0.05)$ were noted

\section{(Figure 4).}

\section{Discussion}

In our large prospective study investigating the burden of multiple respiratory viruses in hospitalized Jordanian children under two years, more than half of the subjects presented with wheezing, and the vast majority 
of subjects had virus detected. The most frequently detected viruses in wheezing subjects in order of highest frequency were RSV, HRV, AdV and HMPV.

Other studies have demonstrated a similar relative proportion of RSV and HRV detected in subjects hospitalized with wheezing illness; in a Japanese study of children under three years hospitalized with lower respiratory tract infections, among wheezing subjects, $33 \%$ had RSV, $14 \% \mathrm{HRV}$, and $2 \% \mathrm{HMPV}^{20}$. In a 4-year study of infants < 12 months hospitalized with bronchiolitis in Greece, $79 \%$ had RSV, $17 \%$ HRV, $2 \%$ HMPV, $10 \%$ influenza, $3 \%$ coronaviruses, and $1 \% \mathrm{PIV}^{21}$. In contrast with our cohort, a Finnish cohort of children 3 months-16 years (median age 2 years) admitted with acute wheezing, they detected a lower frequency of subjects with RSV (14\%), but more subjects had HRV (27\%) and $9 \%$ had HMPV. The older cohort may explain more HRV detected compared with RSV, as studies have shown that HRV-associated LRTI becomes more common as children age ${ }^{21}$. This study also excluded children with severe wheezing and ICU treatment, but noted that RSV-positive children tend to have more severe manifestations of LRTIs than those with $\mathrm{HRV}^{21}$.

We also found that RSV and HMPV were detected in a significantly higher percentage of subjects with wheezing than in those without wheezing, and this persisted after adjusting for multiple variables. Supporting our findings, RSV has been reported to be the leading cause of LRTIs requiring hospitalization in young infants $^{3,5,22,23}$. HMPV has also been identified as an important pathogen in upper and lower respiratory tract infections ${ }^{8}$. Studies of children with acute LRTIs reported HMPV frequency similar to our cohort, ranging from 2-13\%3,5,10,20,21,24, including hospitalized children in the Middle East ${ }^{17}$. Given that HMPV resembles RSV infection cytopathologically, clinically and epidemiologically, it is not surprising that it was found to be an important contributor to wheezing illness in our study.

While HRV was identified in a significant proportion of young, hospitalized patients in our study, we did not observe a significant difference in HRV among those with wheezing versus those without wheezing. Possible reasons for this observation include the fact that the median age of our wheezing subjects was five months, a minority had atopic mothers, relatively few of the children themselves were atopic, and all patients presented with severe symptoms requiring hospitalization. The prevalence of HRV in patients with wheezing and/or bronchiolitis increases with age and is more common in children with recurrent wheezing ${ }^{2,7,21}$. A Finnish study ${ }^{7}$ of hospitalized children found that those with HRV infection were older than those with RSV infection; this is supported by our finding that RSV was detected more frequently in younger subjects with wheezing than older subjects. Studies have also shown that HRV-associated wheezing is observed more frequently in atopic children ${ }^{7,25}$ and that maternal atopic asthma is a risk factor for HRV-associated wheezing ${ }^{7}$. An outpatient study of patients with an atopic parent found that HRV was the most frequently detected infection in LRTIs (40.7\%) and contributed to three times the number of LRTI and wheezing LRTIs than $\mathrm{RSV}^{3}$. Our young cohort with less predominant personal and parental atopic history may explain the predominant effect of RSV (and not HRV) on wheezing.

In our cohort, personal history of RAD or family history of asthma were associated with higher OR of wheezing, but maternal atopy and asthma were not associated with wheezing. According to the Tucson Children's Respiratory Study, there are four patterns of wheezing in early childhood: never wheeze, transient early wheeze, late-onset wheeze and persistent wheeze ${ }^{26}$. Although the current study was not a longitudinal study that would allow us to accurately determine the ultimate wheezing phenotype of our subjects, since most children who wheeze before two years of age do not develop recurrent wheeze or asthma later in childhood $^{2,27}$, it is likely that a significant proportion of children in our cohort were of the transient early wheeze phenotype. Such children do not tend to have history of maternal asthma or personal history of atopy $^{2,26}$. Alternatively, a potential impact of maternal asthma on development of childhood wheeze may not have been detected due to the low frequency of maternal asthma in our cohort. A follow-up study of these children could provide insight regarding whether or not they developed asthma.

This study has both strengths and limitations. Given the large cohort, we were able to compare differences among multiple viruses and the association of wheezing. However, since this was not a longitudinal study and there was no follow-up of subjects to determine if they developed recurrent wheezing or asthma beyond the 
age of five, we are unable to directly ascertain the link between virus-induced wheezing and recurrent wheeze or asthma. While epidemiological studies are often limited by difficulty in accurately diagnosing wheezing in young children, particularly through clinical assessment by parents ${ }^{28}$, in our study wheezing was diagnosed by both clinical assessment and physical exam by a physician, making the diagnosis more reliable. This study investigated wheezing illness specifically in hospitalized children, which provides useful information for patients with potentially more severe disease. This is a year-round surveillance study conducted over a period of three years, and therefore allows us to capture viruses during several seasons. It also is one of the few studies in Jordan/Middle East that used highly a sensitive molecular diagnostic technique for detection of viruses in such a large cohort of hospitalized patients ${ }^{29}$.

In summary, this investigation demonstrates that wheezing in children under two years is associated with RSV and HMPV. Although HRV is a known important risk factor for wheezing, particularly in older children with atopic parental or personal history, an association between HRV and wheezing was not seen in our cohort. This study provides useful data on the burden of respiratory viruses with clinical and demographic factors for wheezing illness. These data can be used to design future longitudinal studies to evaluate the association between early childhood viral acute respiratory infections and recurrent wheezing later in childhood, and to determine which virus interventions, such as vaccination or antiviral therapy that would be most effective at preventing development of wheezing illness.

\section{Acknowledgment}

We thank our research recruiters: Hanan Amin, Amani Altaber, Hana'a Khalaf, Isra'a Kharbat, Darin Yasin, Shireen Issa, and Nurse Sabah Gharbli, and the families who participated in this study. The authors do not have any potential conflicts of interest to disclose

\section{References}

1. Loudon R, Murphy RL, Jr. Lung sounds. Am Rev Respir Dis. 1984;130(4):663-673.

2. Inoue Y, Shimojo N. Epidemiology of virus-induced wheezing/asthma in children. Frontiers in microbiology. 2013;4:391.

3. Kusel MM, de Klerk NH, Holt PG, Kebadze T, Johnston SL, Sly PD. Role of respiratory viruses in acute upper and lower respiratory tract illness in the first year of life: a birth cohort study. The Pediatric infectious disease journal.2006;25(8):680-686.

4. Tenero L, Piazza M, Piacentini G. Recurrent wheezing in children. Translational pediatrics.2016;5(1):31.

5. Mansbach JM, Piedra PA, Teach SJ, et al. Prospective multicenter study of viral etiology and hospital length of stay in children with severe bronchiolitis. Archives of pediatrics $\mathscr{E}$ adolescent medicine. 2012;166(8):700-706.

6. Jackson DJ, Gangnon RE, Evans MD, et al. Wheezing rhinovirus illnesses in early life predict asthma development in high-risk children. American journal of respiratory and critical care medicine. 2008;178(7):667672 .

7. Korppi M, Kotaniemi-Syrjanen A, Waris M, Vainionpaa R, Reijonen TM. Rhinovirus-associated wheezing in infancy: comparison with respiratory syncytial virus bronchiolitis. The Pediatric infectious disease journal. 2004;23(11):995-999.

8. van den Hoogen BG, de Jong JC, Groen J, et al. A newly discovered human pneumovirus isolated from young children with respiratory tract disease. Nature medicine.2001;7(6):719-724.

9. Al-Rousan HO, Meqdam MM, Alkhateeb A, Al-Shorman A, Qaisy LM, Al-Moqbel MS. Human bocavirus in Jordan: prevalence and clinical symptoms in hospitalised paediatric patients and molecular virus characterisation. Singap Med J.2011;52(5):365-369. 
10. Qaisy LM, Meqdam MM, Alkhateeb A, Al-Shorman A, Al-Rousan HO, Al-Mogbel MS. Human metapneumovirus in Jordan: prevalence and clinical symptoms in hospitalized pediatric patients and molecular virus characterization. Diagnostic microbiology and infectious disease. 2012;74(3):288-291.

11. Khuri-Bulos N, Lawrence L, Piya B, et al. Severe outcomes associated with respiratory viruses in newborns and infants: a prospective viral surveillance study in Jordan.BMJ Open. 2018;8(5):e021898.

12. Flohr C, Williams HC. Childhood eczema according to the International Study of Asthma and Allergies in Childhood (ISAAC) questionnaire tool-response to Czarnobilska et al.J Eur Acad Dermatol Venereol. 2011;25(8):993-994; author reply 992-993.

13. Maziak W. The global epidemic of waterpipe smoking. Addictive behaviors. 2011;36(1-2):1-5.

14. Harris PA, Taylor R, Thielke R, Payne J, Gonzalez N, Conde JG. Research electronic data capture (REDCap)-a metadata-driven methodology and workflow process for providing translational research informatics support. J Biomed Inform. 2009;42(2):377-381.

15. Mentel R, Wegner U, Bruns R, Gurtler L. Real-time PCR to improve the diagnosis of respiratory syncytial virus infection. Journal of medical microbiology.2003;52(Pt 10):893-896.

16. Lu X, Holloway B, Dare RK, et al. Real-time reverse transcription-PCR assay for comprehensive detection of human rhinoviruses. J Clin Microbiol. 2008;46(2):533-539.

17. Ali SA, Williams JV, Chen Q, et al. Human metapneumovirus in hospitalized children in Amman, Jordan.J Med Virol. 2010;82(6):1012-1016.

18. Mehlmann M, Bonner AB, Williams JV, et al. Comparison of the MChip to viral culture, reverse transcription-PCR, and the QuickVue influenza $\mathrm{A}+\mathrm{B}$ test for rapid diagnosis of influenza. J Clin Microbiol. 2007;45(4):1234-1237.

19. Khuri-Bulos N, Payne DC, Lu X, et al. Middle East respiratory syndrome coronavirus not detected in children hospitalized with acute respiratory illness in Amman, Jordan, March 2010 to September 2012. Clin Microbiol Infect. 2013.

20. Takeyama A, Hashimoto K, Sato M, et al. Clinical and epidemiologic factors related to subsequent wheezing after virus-induced lower respiratory tract infections in hospitalized pediatric patients younger than 3 years. European journal of pediatrics. 2014.

21. Miller EK, Gebretsadik T, Carroll KN, et al. Viral etiologies of infant bronchiolitis, croup and upper respiratory illness during 4 consecutive years. Pediatr Infect Dis J. 2013;32(9):950-955.

22. Carlsen KH, Orstavik I, Halvorsen K. Viral infections of the respiratory tract in hospitalized children. A study from Oslo during a 90 months' period. Acta paediatrica Scandinavica. 1983;72(1):53-58.

23. Jartti T, Lehtinen P, Vuorinen T, et al. Respiratory picornaviruses and respiratory syncytial virus as causative agents of acute expiratory wheezing in children.Emerging infectious diseases. 2004;10(6):1095-1101.

24. Jartti T, van den Hoogen B, Garofalo RP, Osterhaus AD, Ruuskanen O. Metapneumovirus and acute wheezing in children. Lancet. 2002;360(9343):1393-1394.

25. Jackson DJ, Evans MD, Gangnon RE, et al. Evidence for a causal relationship between allergic sensitization and rhinovirus wheezing in early life. American journal of respiratory and critical care medicine. 2012;185(3):281-285.

26. Martinez FD, Wright AL, Taussig LM, Holberg CJ, Halonen M, Morgan WJ. Asthma and wheezing in the first six years of life. The Group Health Medical Associates. The New England journal of medicine. 1995;332(3):133-138.

27. Karaman O, Uguz A, Uzuner N. Risk factors in wheezing infants. Pediatrics international : official journal of the Japan Pediatric Society. 1999;41(2):147-150. 
28. Bisgaard H, Bonnelykke K. Long-term studies of the natural history of asthma in childhood. The Journal of allergy and clinical immunology.2010;126(2):187-197; quiz 198-189.

29. Khuri-Bulos N, Williams JV, Shehabi AA, et al. Burden of respiratory syncytial virus in hospitalized infants and young children in Amman, Jordan. Scand J Infect Dis.2010;42(5):368-374.

Table 1. Demographic and clinical characteristics of wheezing vs. non-wheezing children

\begin{tabular}{|c|c|c|c|}
\hline & Wheezing $\mathrm{n}=1757$ & No wheezing $\mathrm{n}=1411$ & $\mathrm{P}$ value \\
\hline $\begin{array}{l}\text { Age, months, mean } \\
\pm \text { SD }\end{array}$ & $6.7 \pm 5.6$ & $4.6 \pm 5.3$ & $<0.001^{+}$ \\
\hline $\begin{array}{l}\text { Age, months, median } \\
\text { [IQR] }\end{array}$ & $5[2.4-9.6]$ & $2.1[1.2-6.3]$ & \\
\hline Male & $1075(61.2 \%)$ & $837(59.3 \%)$ & $0.286^{\S}$ \\
\hline $\begin{array}{l}\text { History of or } \\
\text { current } \\
\text { breastfeeding }\end{array}$ & $1452(82.6 \%)$ & $1209(85.7 \%)$ & $0.020^{\S}$ \\
\hline Smoke exposure & $1364(77.6 \%)$ & $1061(75.2 \%)$ & $0.108^{\S}$ \\
\hline CS delivery & $483(27.5 \%)$ & $410(29.1 \%)$ & $0.330^{\S}$ \\
\hline $\begin{array}{l}\text { Birthweight, kg, mean } \\
\pm \mathrm{SD}\end{array}$ & $2.98 \pm 0.7$ & $2.96 \pm 0.7$ & $0.508^{+}$ \\
\hline Prematurity & $252(14.3 \%)$ & $198(14 \%)$ & $0.804^{\S}$ \\
\hline $\begin{array}{l}\text { Underlying Medical } \\
\text { Conditions }\end{array}$ & $208(11.8 \%)$ & $112(7.9 \%)$ & $<0.001^{\S}$ \\
\hline $\begin{array}{l}\text { Child history of } \\
\text { atopy }\end{array}$ & $145 / 1756(8.3 \%)$ & $53 / 1408(3.8 \%)$ & $<0.001^{\S}$ \\
\hline $\begin{array}{l}\text { Reactive Airway } \\
\text { Disease }\end{array}$ & $68 / 1756(3.9 \%)$ & $11 / 1408(0.8 \%)$ & $<0.001^{\S}$ \\
\hline Eczema & $12(0.7 \%)$ & $6(0.4 \%)$ & $0.339^{\S}$ \\
\hline Allergic rhinitis & $80 / 1755(4.6 \%)$ & $39 / 1409(2.8 \%)$ & $0.009^{\S}$ \\
\hline $\begin{array}{l}\text { Mother history of } \\
\text { atopy }\end{array}$ & $242(13.8 \%)$ & $191(13.5 \%)$ & $0.847^{\S}$ \\
\hline Asthma & $53(3 \%)$ & $38(2.7 \%)$ & $0.588^{\S}$ \\
\hline Eczema & $67(3.8 \%)$ & $55(3.9 \%)$ & $0.902^{\S}$ \\
\hline Allergies & $150(8.5 \%)$ & $116(8.2 \%)$ & $0.750^{\S}$ \\
\hline $\begin{array}{l}\text { Family history of } \\
\text { asthma }\end{array}$ & $435(24.8 \%)$ & $285(20.2 \%)$ & $0.002^{\S}$ \\
\hline Oxygen therapy & $647(37.2 \%)$ & $366(26.2 \%)$ & $<0.001^{\S}$ \\
\hline Mechanical Ventilation & $69(4 \%)$ & $42(3 . \%)$ & $0.149^{\S}$ \\
\hline Intensive care unit & $168(9.6 \%)$ & $116(8.2 \%)$ & $0.189^{\S}$ \\
\hline $\begin{array}{l}\text { Length-of-stay, } \\
\text { days, mean } \pm \text { SD }\end{array}$ & $5.2 \pm 3.9$ & $6.1 \pm 4.2$ & $<0.001^{+}$ \\
\hline
\end{tabular}

n= number; CS: cesarean section; Kg: kilogram; SD: standard deviation: IQR: interquartile range

Categorial Data are in $\mathrm{n}(\%)$, Continuous Data are in Mean $\pm \mathrm{SD}$, Median [IQR]

$\S$ Pearson's Chi-Squared, + T-test

Figure Legends

Figure 1. Study Enrollment Diagram and Results of Respiratory Specimens and Wheezing Frequency. 
Figure 2. Comparison of Wheezing and No Wheezing Groups, By Frequency of Virus-Detection.

Figure 3. Virus Frequency by Age Among Virus-Positive Wheezing Subjects

Figure 4. Multivariable Logistic Regression Evaluate the Association Between Variables and Wheezing.
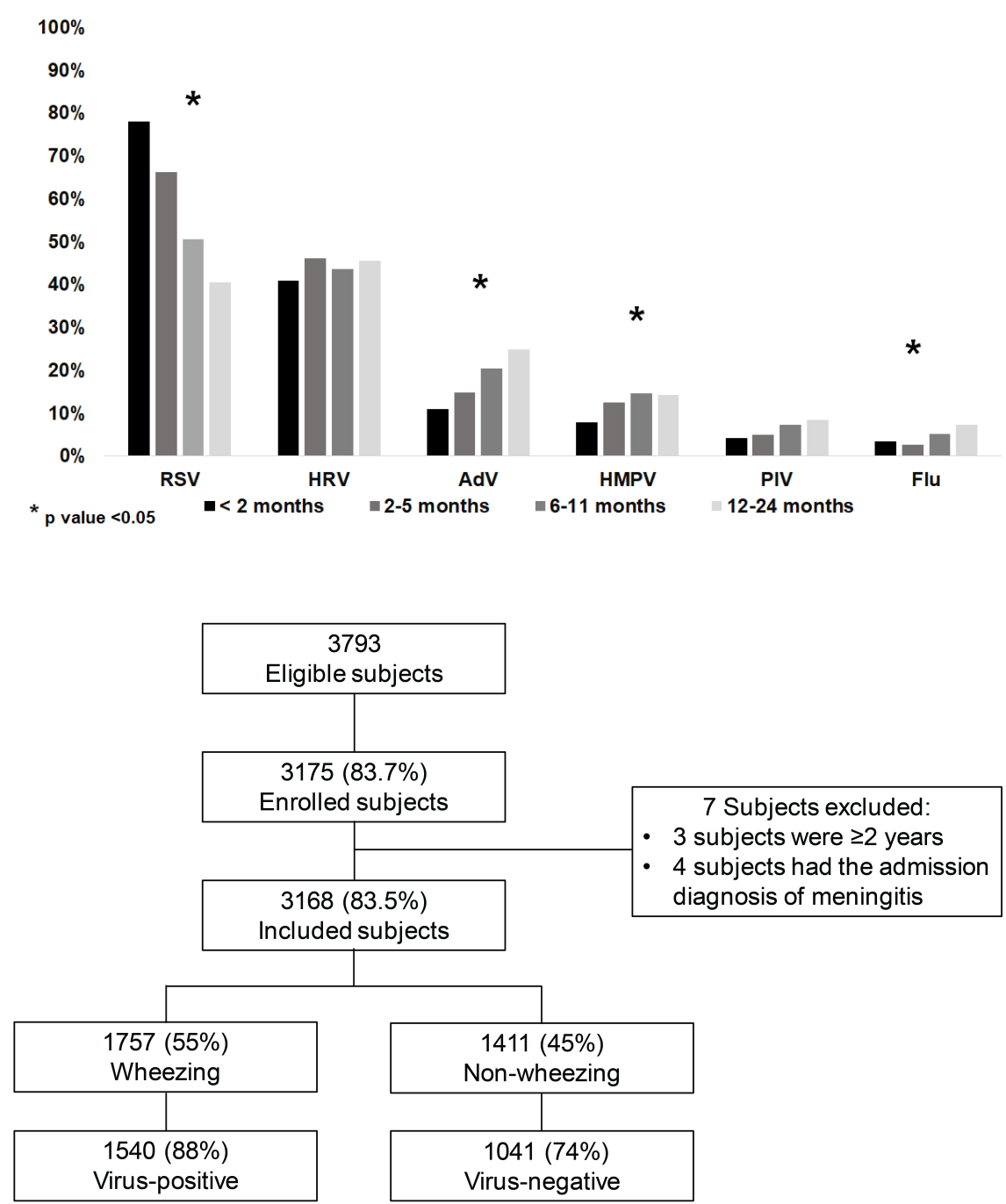


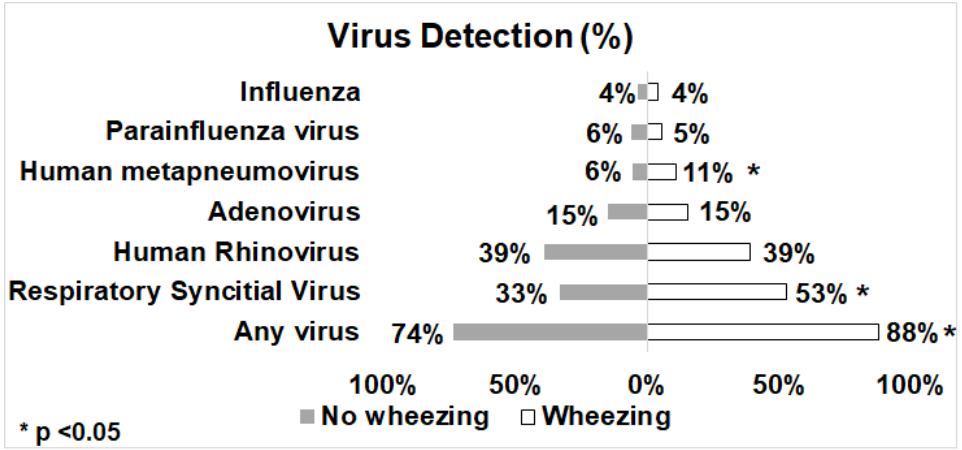

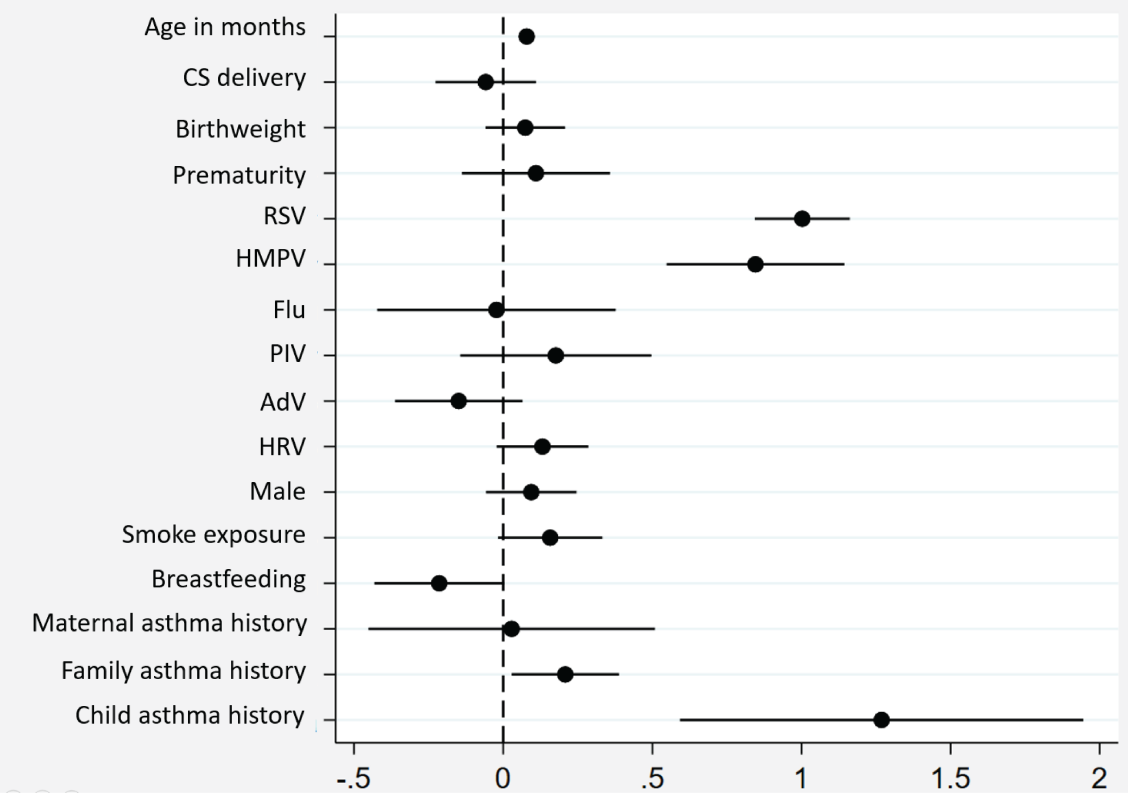

